\title{
Performance Evaluation of Different Short Path Algorithms to Improve Oil-Gas Pipelines
}

\author{
Nabeel Naeem Almaalei ${ }^{1}$, Siti Noor Asyikin Mohd Razali ${ }^{2}$ \\ Department of Mathematics and Statistics \\ Faculty of Applied Sciences and Technology \\ University Tun Hussein Onn Malaysia \\ Pagoh Education Hub \\ 84600 Pagoh, Johor
}

\begin{abstract}
The oil-gas pipeline is a complicated and expensive system in terms of construction, control, materials, monitoring, and maintenance which includes economic, social and environmental hazards. As a case study of Iraq, the system of pipelines is above the ground and is liable to disasters that may produce an environmental tragedy as well as the loss of life and money. Hence, this article presents a performance evaluation of different short path algorithms to improve oil-gas pipelines. The chosen algorithms in this paper were Parallel Short Path Algorithm (PSPA), Ant Colony Optimization (ACO) algorithm and Genetic Algorithm (GA). The main performance metric is the cost of the pipelines. Simulation trials were performed using the MATLAB program for the chosen algorithms. The performance comparison showed that the lowest cost of laying oil and gas pipelines was by applying the GA algorithm when the number of wells was set to 50-600. Conversely, the PSPA algorithm showed the best performance in terms of required implementation time for all scenarios. Besides, PSPA appeared to have acceptable performance in terms of the cost of the pipeline when the number of wells was arranged between50-600. Furthermore, PSPA showed the best performance for 700 and 840 wells in terms of the cost of laying the oil and gas pipelines compared to ACO and GA. It should be noted that the ACO algorithm showed medium performance in terms of the cost of laying oil and gas pipelines compared to PSPA and GA.
\end{abstract}

Keywords-PSPA; ACO; GA; Oil-Gas pipeline; performance; cost; short path

\section{INTRODUCTION}

Oil is a crucial source of energy. It is used in many industries, transportations and electricity supplies. Oil is transferred from the oil fields to the main stations (oil refineries) that require a network of pipes, which include valves and pipes of different diameters and pumping stations. When oil passes through the pipelines, there occurs a loss of $3 \%$ of the total oil due to the evaporation process since the oil pipelines carry large quantities of oil. The oil pipelines are used to transport (import and export) petroleum products between different cities around the world with about 17.95 million barrels per day [1]. This has led to the development of large and extensive design as well as operations of pipelines which have become more complex in recent years [2]. The main issue discussed in this proposal is in applying the proposed algorithm to find the shortest path that links the oil wells with the gathering facilities, hence obtaining the least cost for the work of the oil-gas pipeline network.

The optimization network of pipelines for gathering and transporting oil and gas should include the location, parameter, and topology. The topology structure mentioned here is the multilevel star (MS), multistage star-ring (MSR) and the multistage star-tree (MST). In the past few years, some optimization algorithms have been used to improve the oil-gas pipeline network such as the ant colony optimization (ACO) algorithm, the particle swarm (PS) algorithm, artificial neural network (ANN) algorithm, and the Genetic algorithm (GA). Many researchers studied the improvement of pipeline networks since the early 1970s. For example, Gabriele (1977) [3] proposed the Steiner algorithm, whereas Edgar (1978) used a generalized reduced gradient method for the first time. Also, Simposon et al. (1990) [4] provided a GA to the optimization design, while Shuwen (1998) proposed the Kruskal algorithm as well as the Prime algorithm. On the other hand, in prior work [5], the authors used a genetic algorithm to supply the natural gas distribution network, while in the same year, Jiancheng in [6], who was the first researcher to use GA in the oil-gas pipeline network, used ANN to determine the multilevel star network. Furthermore, Jianjun combined the Simulated Annealing algorithm (SAN) and GA to solve the star network topology. In a previous work [7], the paper presented an optimization algorithm fitted for the oil-gas gathering network which considered several obstacles.

Fig. 1 shows the map of numerous oil fields in Iraq. Many oil fields are scattered around southern and northern Iraq, where the city of Basra is one of the largest cities in Iraq containing oil fields. It consists of 530 geological formations which indicate the existence of a large quantity of oil. One hundred and fifteen oil fields were drilled, including 27 fields proven to contain a large oil reserve estimated at 65 billion barrels. Fifty-nine percent of the total oil reserves were distributed to other fields including the North Rumaila, South Rumaila, Majnoon, Zubair, River Omar, West Qurna, AlTuba, Al-Sabah, Al-Halafiyya, and Abu-Gharb and AlBazarkan fields [9]. 


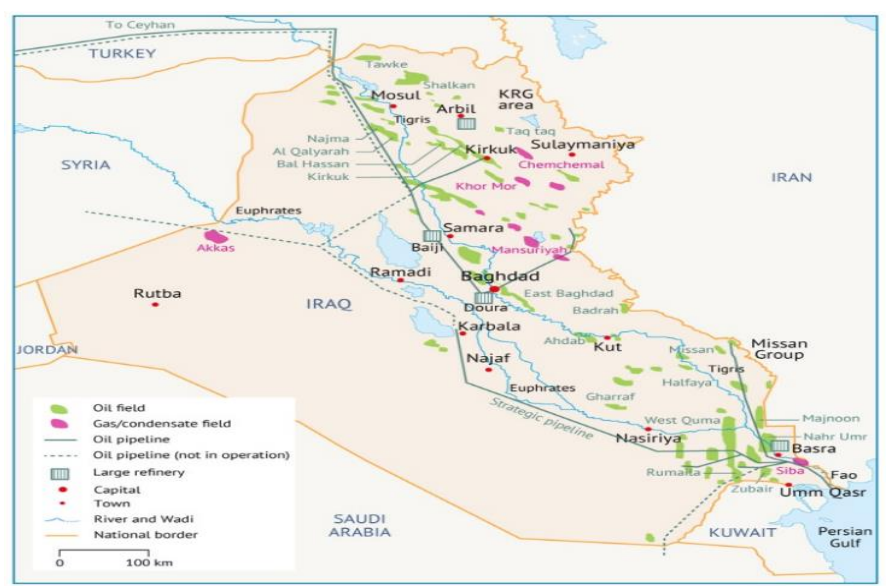

Fig. 1. Map of Numerous oil Fields in Iraq. [8].

\section{LITERATURE REVIEW}

\section{A. Oil-Gas Pipeline Network}

The transportation of solid, liquid and gaseous substances is crucial and necessary for humans. The ancient Chinese began this by using the stems of Bamboo plants to transport liquids and water up to three inches in diameter, using sloping passages to transport liquids. The idea of pipelines came from here.

Pipelines are a reliable and safe way to transport products necessary for the national economy of many countries in the world such as water, oil, and gas [10]. Dandy et al. in [11] applied the genetic algorithm and developed a technique to improve the water pipeline networks to find the minimum cost of construction. Concerning oil and gas pipeline networks, they transported and distributed large quantities of oil and gas worldwide. It was considered the safest way to transport because of its limited number of failures. In 1879, in Pennsylvania, the United States built the first oil pipeline with a length of 109 miles and a diameter of 6 inches [12]. Now, there are more than 60 countries with pipeline networks, with a length of more than $2000 \mathrm{~km}$. The United States has the longest pipeline network in the world followed by Russia [13]. Many researchers had discussed the improvement of oil and gas pipeline networks, specifically in the 1970s. Some optimization algorithms such as Prime and Kruskal were proposed by Shuwen in [14] to improve the natural gas networks. The Prime algorithm is a greedy algorithm that finds the minimum extension tree for an unweighted graph. This implies that it finds a subset of the edges that make up a tree including each top of the head every time and adds the least possible connection from the tree to another's top. This algorithm was developed by the computer scientist Edsger W. Dijkstra in 1959 [15]. Therefore, it is also sometimes known as the Dijkstra algorithm. The Kruskal algorithm was also used to solve the same problem.

In 1996, the genetic algorithm technique was developed to improve pipeline networks, and its formulation was continuously improved to solve the problem of spending in New York. The genetic algorithm was considered better than traditional methods of improvement such as dynamic programming methods as well as linear and nonlinear programming [11]. This technique was powerful and was able to create the minimum cost of pipeline network constructions compared to the size of the search area. It was used to improve the water pipeline networks, taking into account the length and size of the pipe in the design to evaluate the target function [4].

Multi-objective optimization methods for the transportation of gas differ fundamentally from individual target improvement methods. On this basis, the technique of improving the ant colony of multipurpose was developed to improve the transportation of gas in pipelines by reducing the consumption of the compressor fuel as well as increasing the production to maximum [16]. The design of the gas pipeline networks was complex where it consisted of pipes and element controls such as pumps and valves. Control of the gas pipeline network required a suitable environment for all elements without violating the physical and operational limitations during the process of transferring gas from the moment the network enters to the moment it exits. In 2011, Ermin et al. [17] presented the article provided a procedure to calculate the appropriate network accessories and optimal cost based on rigorous mathematical programming methods. In 1978, Edgar [18] presented a study of a calculation algorithm to improve the design of the gas pipeline networks by determining the number of compressors, the length and diameter of the pipes, as well as the factors affecting the operation of the storage compressors to reduce the operating costs and capital using two solution techniques. The first method was applied to cases where the capital costs came from a non-zero initial fixed cost plus some horsepower output function, whereas the second method was known as the low gradient method, which is a nonlinear programming algorithm used directly in cases where the capital cost of compressors is a function of the horsepower output but does not contain any initial fixed cost [18].

Marine pipelines transporting oil and gas are exposed to erosion and degradation. It is, therefore, necessary to predict and monitor those pipelines to minimize accidents that may occur as well as optimize the operation. Models for the forecasting and evaluation of the state of oil and gas pipelines were developed using artificial neural network technology (ANN) based on the data provided for three oil and gas pipelines in Qatar. These models were able to predict the state of pipelines by a success rate of $97 \%$ [13].

Crude oil with high viscosity, freezing point, and wax content is usually transported through hot pipelines, known as the hot oil pipeline (HOP), equipped with pumping and heating stations. The cost of fuel and energy for pumping stations and heating ranges from 1-3\% of total energy consumption. On this basis, a model was designed to reduce the cost of the operating power of HOP with the temperature of the output of each heating station and the operation of each pump as variables for improvement. The differential evolution (DE) algorithm was combined with the particle swarm optimization (PSO) algorithm to solve this model, where the optimum operating model saved $17.95 \%$ of the cost of energy needed to transport crude oil to a distance of $2640 \mathrm{~m} 3 / \mathrm{h}$ [5]. 


\section{B. Oil-Gas Parallel Shortest Path Algorithm}

The proposed algorithm in [19] was based on finding the distances between the wells by giving the site (Cartesian coordinates) of each well and then choosing the shortest path that connects the two wells. We take into consideration the obstacles that may be located on the track which leads to finding an alternative path and finally reaching the shortest path connecting all the wells with the main station.

Parallel Shortest Path Algorithm (PSPA)

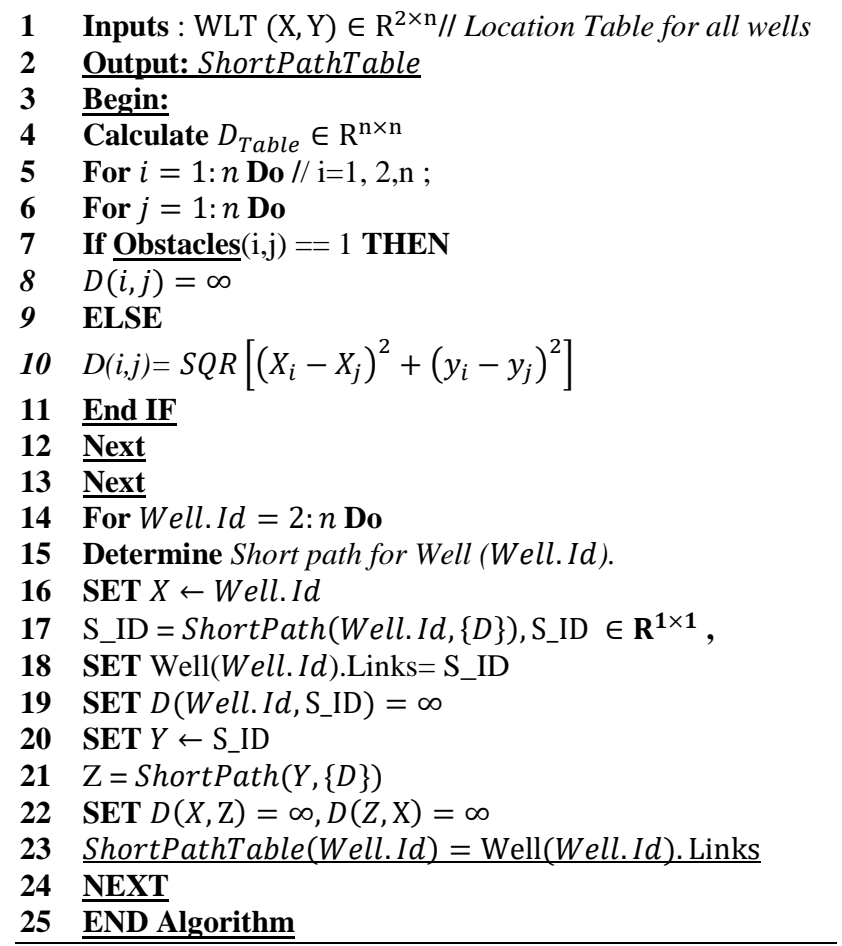

\section{Formulation of ACO Algorithm in a Mathematical Model}

Fig. 2 shows the ACO algorithm flowchart. We have the following mathematical model as given by:

$p_{i j}^{k}=\frac{\left[\tau_{i j}\right]^{\alpha}\left[\zeta_{i j}\right]^{\beta}}{\sum_{z \in \text { allowed } y}\left[\tau_{i j}\right]^{\alpha}\left[\zeta_{i j}\right]^{\beta}}$

where

$k:$ the ant.

$p_{i j}^{k}$ : The ant moving from $i$ to $j$.

$\tau_{i j}$ : The amount of pheromone along the transition from node $i$ to $j$.

$\propto \geq 0$ : The parameter to control the influence of $\tau_{i j}$.

$\zeta_{i j}$ : The desirability of node transition $i j$ (a priori knowledge, typically $1 / d_{i j}$, where $d$ is the distance).

$\beta \geq 1$ : is a parameter to control the influence of $\zeta_{i j}$.

$\tau_{i j}, \zeta_{i j}:$ represents the attractiveness and trail level for the other possible node transitions.
- Pheromone update

When all the ants have completed a solution, the trails are updated by [20]:

$\tau_{i j} \leftarrow(1-\rho) \tau_{i j}+\sum_{k} \Delta \tau_{i j}^{k}$

and

$\Delta \tau_{i j}^{k}=\left\{\frac{Q}{L_{k}}\right.$ if ant uses curve ij in its tour 0 otherwise

where

$L_{k}:$ the cost of the $K_{t h}$ ant's tour.

$Q:$ is a constant

- Discussion variable

The discussion variable is presented as follows:

$\mathrm{V}=\left\{v_{0}, v_{1}, v_{2}, \ldots v_{n}\right\}$. The set of wells (nods) and their number $n$

$\mathrm{E}=\{(i, j): i, j \in V, i \neq j\}$.

$d_{i j}=\sqrt{\left(x_{i}-x_{j}\right)^{2}+\left(y_{i}-y_{j}\right)^{2}} \forall(i, j) \in E$.

$d_{i j}=$ The matrix of distant between wells.

$\mathrm{E}$ : The set of ribs that reaches between the wells (nodes).

$v_{0}$ : The wells from which the path starts.

$\mathrm{C}_{\mathrm{ij}}$ : The cost matrix for moving from the well from $i$ to $j$, (i.e., distance $\left.d_{i j}{ }^{*} \cos t\right)$.

$\mathrm{t}_{\mathrm{ij} \text { : }}$ time of arrival between wells $i$ and $j,\left(\mathrm{t}_{\mathrm{ij}}>0\right)$.

$\mathrm{t}_{\mathrm{i}}$ : time of arrival to well $i$.

// ACO for Oil-Gas Pipeline Wells Problem //

- Input: Location $\left(x_{i}, y_{i}\right), x$ and $y \in R^{1 \times n}$,

$i=1,2,3 \ldots ., n$

- Output : Final cost

- Apply ACO ShortPath (Data) // for all well locations

- Min $_{\text {cost }} \leftarrow$ ACOShortPath (Data) // Estimate the minimum cost / shortest path for all wells locations

- Estimate the final cost based on the Wells problem as the follows

$$
\text { Final }_{\text {cost }}=\text { Min }_{\text {cost }}-L W_{\text {cost }}
$$

where $L W_{\text {cost }}$ is the cost of the last well . //

\section{GA Algorithm}

It is known that the genetic algorithm is a smart algorithm to choose the best solution among a large number of solutions and to make interventions and modifications between these solutions to create a better solution. In general, the genetic algorithm contains several basic steps to resolve various issues that differ in the method of formulation and implementation according to the issue and scope of application. The following is an explanation of the proposed genetic algorithm steps to solve the issue of the shortest path linking the oil-gas wells with the main station. 


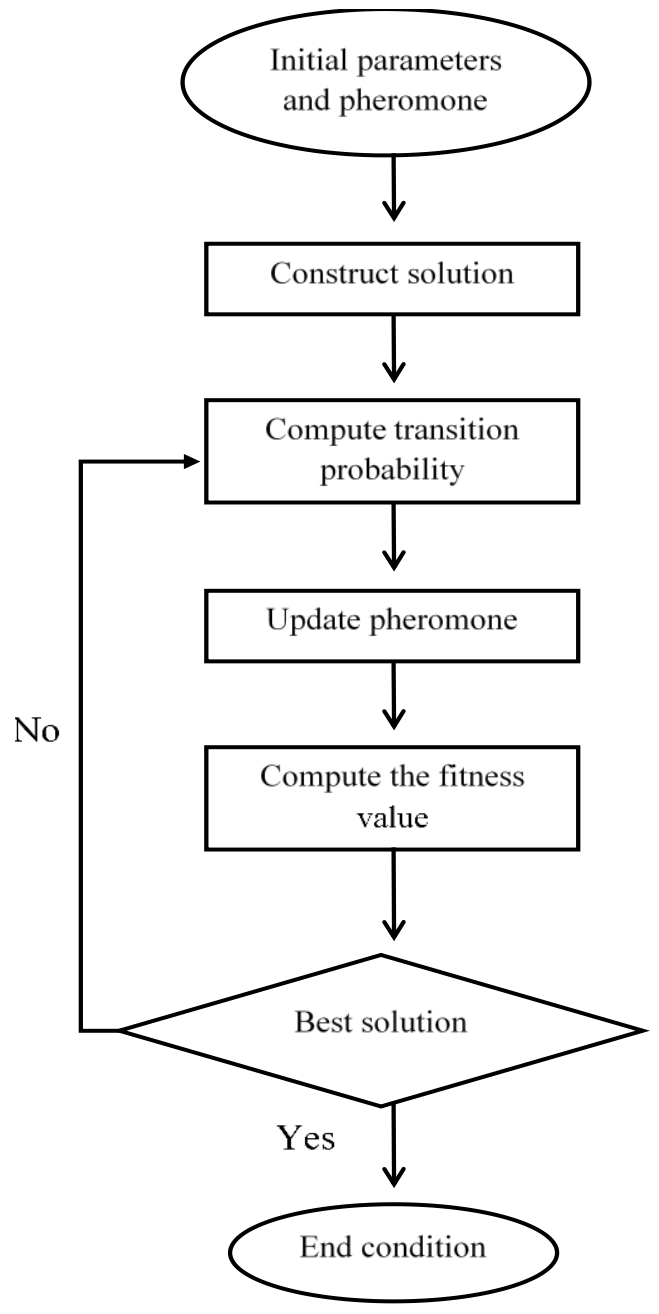

Fig. 2. ACO Algorithm Flowchart [21].

Step 1: (Initial data): This is a reading of the variables' values for the issue of linking oil-gas wells and is a cost or distance matrix. It represents the cost or the distance between the wells.

Step 2: (Generation initial population): The first point is the process of building the primary generation in a random way where several initial chromosomes are created so that each chromosome represents an integrated path. The length of the path is the number of points to pass where each gene of the chromosome represents the location of a well. The figure below shows a chromosome of length $\mathrm{L}$.

Step 3: (Objective function): In this step, the objective function of the issue and each of the generation segments, which represent the sum of the costs or distances, are evaluated.

Step 4: (Selection): All chromosomes of the primary generation are selected as parents. Each chromosome mates with all chromosomes in the primary generation where each mating process produces two new individuals.

Step 5: (Generation): After selecting parent segments, subsequent generations are generated, and the number is specified by the value entered at the beginning of the execution. Fig. 3 illustrates the flow chart for generations.

Step 6: (Crossover): The overlapping process is the selection of two segments. Then, the process of transition is performed to obtain the offspring. The process of creative interference in the issue of the traveling salesman problem differs from other issues because each gene represents the sequence of a city and the city cannot be repeated within the chromosome.

Step 7: (Mutation): In this algorithm, the mutation occurs on the same gene. After configuring the generation segments, the target function for each section of the new generation is calculated in the same way that the initial generation segments were calculated. After configuring the specified number of generations, the execution of the function stops, and the results are evaluated to observe the closeness to decide whether to continue generational formation or to stop if the results are appropriate. The type of proposal is in the method of formulating and applying each step of the proposed algorithm. The chromosome is a complete pathway (each gene represents a city sequence).

\begin{tabular}{|l|l|l|l|l|l|}
\hline Gene1 & Gene2 & Gene3 & $\cdots$ & $\cdots$ & Gene L \\
\hline
\end{tabular}

Fig. 3. Generations Flowchart.

// GA for Oil-Gas Pipeline Wells Problem //

- Input: Location $\left(x_{i}, y_{i}\right), x$ and $y \in R^{1 \times n}, i=$ $1,2,3 \ldots ., n$

- Output : Final cost $_{\text {cos }}$

- Apply GA ShortPath (Data) // for all well locations

- Min $_{\text {cost }} \leftarrow$ GAShortPath (Data) // Estimate the minimum cost / shortest path for all wells locations

- Estimate the final cost based on the Wells problem as the follows

$$
\text { Final }_{\text {cost }}=\text { Min }_{\text {cost }}-L W_{\text {cost }}
$$

where $L W_{\text {cost }}$ is the cost of the last well . //

\section{SimUlation AND RESULTS}

The scope of this study was around the northern Rumaila field, known as the largest oil field in Basra. It was discovered in 1953 with a length of $80 \mathrm{~km}$ and a width of $4 \mathrm{~km}$, where the number of wells found was between $600-840$ oil and gas wells [19]. All simulation experiments reported in this paper applied the model attributes as shown in Table I.

TABLE. I. Simulation Model Attributes ANd Parameters Value

\begin{tabular}{|l|l|l|}
\hline Parameter & Value & Note \\
\hline No. wells & $50-840$ & For all algorithms (PSPA, GA, and ACO ) \\
\hline Pop.Size & $10-50$ & For GA and ACO [23];[25] \\
\hline numIter & 1 e5 & For GA \\
\hline maxIts & 300 & For ACO \\
\hline numAnts & $10-50$ & For ACO [22];[24] \\
\hline beta & 1 & For ACO \\
\hline Q & 1 & For ACO \\
\hline rho & 0.4817 & For ACO \\
\hline q_0 & 0.2770 & For ACO \\
\hline
\end{tabular}


where

numIter : number of iteration for GA Algorithm.

maxIts : number of iteration for ACO Algorithm. numAnts : number of ants for ACO Algorithm.

beta : A parameter which determines the weight of the heuristic function eta

Q : constant that determines how to update tau.

rho : the pheromone evaporation rate.

q_0 : A parameter determining how often we take the route with the best.

TABLE. II. COMPARISON OF DIFFERENT METHODS

\begin{tabular}{|c|c|c|c|c|c|c|}
\hline \multirow{2}{*}{$\begin{array}{l}0 \\
\overline{0} \\
3 \\
\dot{3} \\
\dot{0} \\
\dot{z}\end{array}$} & \multicolumn{2}{|c|}{ PSPA algorithm } & \multicolumn{2}{|c|}{ ACO algorithm } & \multicolumn{2}{|l|}{$\begin{array}{l}\text { GA } \\
\text { algorithm }\end{array}$} \\
\hline & Cost & $\begin{array}{l}\text { Run } \\
\text { Time } \\
\text { (sec.) }\end{array}$ & Cost & $\begin{array}{l}\text { Run } \\
\text { Time } \\
\text { (sec.) }\end{array}$ & Cost & $\begin{array}{l}\text { Run } \\
\text { Time } \\
\text { (sec.) }\end{array}$ \\
\hline 50 & 74626.56 & 1.08 & 69389.00 & 18.45 & 63026.76 & 792.10 \\
\hline 100 & 123310.83 & 1.45 & 122160.47 & 31.65 & 108717.03 & 850.27 \\
\hline 200 & 365176.59 & 2.86 & 316753.14 & 59.07 & 300203.50 & 921.23 \\
\hline 300 & 489842.80 & 4.65 & 383333.61 & 113.59 & 360297.20 & 1013.17 \\
\hline 400 & 526711.57 & 7.42 & 484269.53 & 149.67 & 439977.22 & 1068.95 \\
\hline 500 & 586227.15 & 9.58 & 547907.20 & 200.69 & 539163.68 & 1146.49 \\
\hline 600 & 635362.30 & 12.39 & 634612.61 & 272.91 & 619332.93 & 1259.24 \\
\hline 700 & 693048.53 & 15.84 & 695105.33 & 359.73 & 745123.53 & 1279.89 \\
\hline 840 & 740728.59 & 21.74 & 775026.42 & 459.05 & 903172.01 & 1461.05 \\
\hline
\end{tabular}

Table II and Fig. 4 show the comparison results of different methods. The studied algorithms (PSPA, ACO, and GA algorithms) were applied to determine the short path for various scenarios, where the number of wells was set to 50 , $100,200,400,500,600,700$ and 840 . From the results, it was clear that the lowest cost of laying oil and gas pipelines was by applying the GA algorithm when the number of wells was set to 50-600. Conversely, the PSPA algorithm showed the best performance in terms of required implementation time for all scenarios. Besides, PSPA appeared to have acceptable performance in terms of the cost of the pipeline when the number of wells was arranged between 50 and 840 . Furthermore, PSPA showed the best performance for 700 and 840 wells in terms of the cost of laying oil and gas pipelines compared to ACO and GA. It should be noted that the ACO algorithm showed medium performance in terms of the cost of laying oil and gas pipelines compared to PSPA and GA.

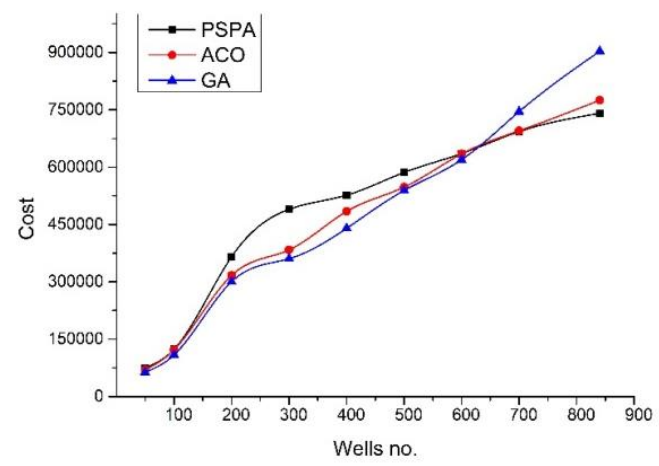

Fig. 4. The Comparison of different Methods in Terms of Cost.

\section{CONCLUSIONS}

This paper presents a performance evaluation of different short path algorithms to improve oil-gas pipelines. The chosen algorithms in this paper are PSPA, ACO, and GA algorithms where the main performance metric is the cost of pipelines. The performance comparison showed that the lowest cost of laying oil and gas pipelines was by applying the GA algorithm when the number of wells was set to 50-840. Conversely, the PSPA algorithm showed the best performance in terms of required implementation time for all scenarios. Besides, PSPA appeared to have acceptable performance in terms of the cost of the pipeline when the number of wells was between50-840. Furthermore, the PSPA algorithm showed better performance for 700 and 840 wells in terms of the cost of laying oil and gas pipelines compared to $\mathrm{ACO}$ and GA. Note that the ACO algorithm showed middle performance in terms of the cost of laying oil and gas pipelines compared to PSPA and GA.

\section{ACKNOWLEDGMENT}

Authors would like to thank Universiti Tun Hussein Onn Malaysia (UTHM) for kindly providing us with the internal funding. This work is also supported by the Research Management Centre (RMC), Universiti Tun Hussein Onn (UTHM) under the TIER 1 grant number H075.

\section{REFERENCES}

[1] BP, "Statistical Review of World Energy 2014," Br. Pet., no. June, pp. $1-45,2013$.

[2] H. R. Zhang, Y. T. Liang, Q. Xiao, M. Y. Wu, and Q. Shao, "Supplybased optimal scheduling of oil product pipelines," Pet. Sci., vol. 13, no. 2, pp. 355-367, 2016.

[3] G. A. Gabriele, "The Generalized Reduced Gradient Method: A Reliable Tool for Optimal Design,” no. May 1977, pp. 394-400, 1977.

[4] A.R. Simposon, L. Murphy, and G. Dandy, "Pipe Network Optimisation Using Genetic Algorithms by University of Adelaide South Australia 5001 Paper to be presented at the ASCE Water Resources Planning and Management Conference Seattle , Washington Using Genetic Algorithms ." Proc, ASCE. Water Resources Planning and Management Division," no. May, 1993.

[5] J. Zhou, G. Liang, T. Deng, and J. Gong, "Route Optimization of Pipeline in Gas-Liquid Two-Phase Flow Based on Genetic Algorithm," Int. J. Chem. Eng., vol. 2017, 2017.

[6] Jiancheng, "Jiancheng, L., 2001. The Topology Optimization Design of Oil-Gas Gathering and Transportation [J]. Petroleum Planning \& Engineering, 6," p. 2001, 2002.

[7] Zhang.Z, The Optimization and Programming of Oilfield Surface Gathering Network [D], China University of Petroleum (Esat China), 2011, pp.1-15.

[8] "A comparison of burial, maturity and temperature histories of selected wells in southern Iraq," no. October, 2017.

[9] Basrah oil co., "boc.oil.gov.iq.pdf," 2016. [Online]. Available: http://www.boc.oil.gov.iq/index.php?name=Pages\&op=page \&pid=108. [Accessed: 08-Mar-2016].

[10] W. Dawotola, P. H. A. J. van Gelder, and J. Vrijling, "Risk Assessment of Petroleum Pipelines using a combined Analytical Hierarchy Process Fault Tree analysis," Proc. 7th Int. Probabilistic Work. Fac. Civ. Eng. Geosci. Delft Univ. Technol. Stevinweg 1, 2628CN Delft, Netherlands, vol. 13, no. 2006, pp. 491-501, 2009.

[11] G. C. Dandy, A. R. Simpson, and L. J. Murphy, "An improved genetic algorithm for pipe network optimization," Water Resour. Res., vol. 32, no. 2, pp. 449-458, 1996.

[12] R. Eberhart and J. Kennedy, "A new optimizer using particle swarm theory," MHS'95. Proc. Sixth Int. Symp. Micro Mach. Hum. Sci., pp. $39-43$. 
[13] M. S. El-Abbasy, A. Senouci, T. Zayed, F. Mirahadi, and L. Parvizsedghy, "Artificial neural network models for predicting condition of offshore oil and gas pipelines," Autom. Constr., vol. 45, pp. 50-65, 2014.

[14] Shuwen, Zhang and R.Meng, Pileline layout Optimization of Large Gas Field Gathering [J], Petroleum Planning \& Design, 1998.

[15] E. W. Dijkstra, "Dijkstra.Pptx," vol. 271, pp. 269-271, 1959.

[16] A. K. Arya and S. Honwad, "Multiobjective optimization of a gas pipeline network: an ant colony approach," J. Pet. Explor. Prod. Technol., no. 123456789, 2017.

[17] Ermin et al., "Gas Network Topology Optimization for Upcoming Market Requirements 1," vol. 9, no. March, 2011.

[18] Edgar, T.F.Himmelblau D.M, Bickel T.C, Optimal Design of Gas Transmission Networks[J].Society of Petroleum Engineering Journal.SPE Number, 4:6031,1978.

[19] Almaalei, Nabeel Naeem Hasan, Siti Noor Asyikin Mohd Razali, and Nayef Abdulwahab Mohammed Alduais. "An efficient algorithm to improve oil-gas pipelines." International Journal of Engineering \& Technology 7, no. 4 (2018): 5412-5418.
[20] Ning, J., Zhang, Q., Zhang, C., \& Zhang, B. (2018). A best-pathupdating information-guided ant colony optimization algorithm. Information Sciences, 433-434, 142-162. https://doi.org/10.1016/ j.ins.2017.12.047

[21] Yoo, K. S., \& Han, S. Y. (2013). A modified ant colony optimization algorithm for dynamic topology optimization. Computers and Structures, 123, 68-78. https://doi.org/10.1016/j.compstruc.2013.04.012.

[22] David Renfrew. (2009). Traffic Signal Control with Ant Colony Optimization.

[23] Gonen, B. (2011). Genetic Algorithm Finding the Shortest Path in Networks. International Conference on Genetic and Evolutionary Methods, (January 2011), 1-4.

[24] Li, J., Sun, S., Huang, Y., \& Wang, N. (2010). Research into selfadaptive hybrid ant colony algorithm based on flow control. Proceedings - 2010 2nd International Workshop on Intelligent Systems and Applications, ISA 2010, (978). https://doi.org/10.1109/IWISA. 2010.5473446.

[25] Scrucca, L. (2017). On some extensions to GA package: Hybrid optimisation, parallelisation and islands evolution. R Journal, 9(1), 187206. https://doi.org/10.32614/rj-2017-008. 